\title{
Bistatic Synthetic Aperture Radar Imaging for Arbitrary Flight Trajectories
}

\author{
Can Evren Yarman, Member, IEEE, Birsen Yazıcı, Senior Member, IEEE, and Margaret Cheney, Member, IEEE
}

\begin{abstract}
In this paper, we present an analytic, filtered backprojection (FBP) type inversion method for bistatic synthetic aperture radar (BISAR). We consider a BISAR system where a scene of interest is illuminated by electromagnetic waves that are transmitted, at known times, from positions along an arbitrary, but known, flight trajectory and the scattered waves are measured from positions along a different flight trajectory which is also arbitrary, but known. We assume a single-scattering model for the radar data, and we assume that the ground topography is known but not necessarily flat. We use microlocal analysis to develop the FBP-type reconstruction method. We analyze the computational complexity of the numerical implementation of the method and present numerical simulations to demonstrate its performance.
\end{abstract}

Index Terms-Bistatic, filtered backprojection, microlocal analysis, radar, synthetic aperture imaging.

\section{INTRODUCTION}

$\mathbf{I}$ $\mathrm{N}$ synthetic aperture radar (SAR) imaging, a scene of interest is illuminated by electromagnetic waves that are transmitted from an antenna mounted on a plane or satellite. The aim is to reconstruct an image of the scene from the measurement of the scattered waves.

Unlike its monostatic counterpart, where transmitter and receiver antennas are co-located (Fig. 1), in bistatic SAR (BISAR) [1], transmitter and receiver antennas are located on separate platforms (Fig. 2). This allows the transmitter and its heavy power supply to be flown on a platform different from that of the cheap, expendable receiver. Also, some of the electronic countermeasures that have been devised to thwart monostatic configurations are less effective against bistatic systems [2], [3]. Finally, bistatic measurements can provide better ability to distinguish targets from clutter [4].

Manuscript received January 3, 2007; revised September 26, 2007. This work was supported by the Air Force Office of Scientific Research under the agreements FA9550-04-1-0223 and FA9550-06-1-0017. Because of this support, the U.S. Government is authorized to reproduce and distribute reprints for Governmental purposes notwithstanding any copyright notation thereon. The views and conclusions contained herein are those of the authors and should not be interpreted as necessarily representing the official policies or endorsements, either expressed or implied, of the Air Force Research Laboratory or the U.S. Government. The associate editor coordinating the review of this manuscript and approving it for publication was Dr. Jacques Blanc-Talon.

C. E. Yarman is with the Houston Technology Center, WesternGeco-Schlumberger, Houston, TX 77042 USA (e-mail: yarman@ecse.rpi.edu).

B. Yazıcı is with the Electrical, Computer, and System Engineering Department, Rensselaer Polytechnic Institute, Troy, NY 12180 USA (e-mail: yazici@ecse.rpi.edu).

M. Cheney is with the Department of Mathematical Sciences, Rensselaer Polytechnic Institute, Troy, NY 12180 USA (e-mail: cheney@ @pi.edu).

Digital Object Identifier 10.1109/TIP.2007.911812

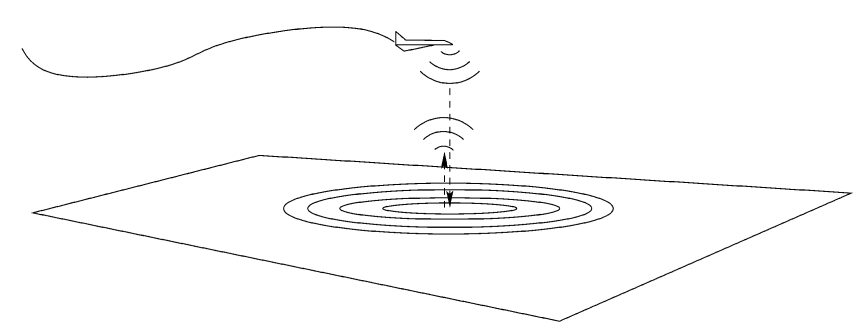

Fig. 1. Acquisition geometry for monostatic SAR.

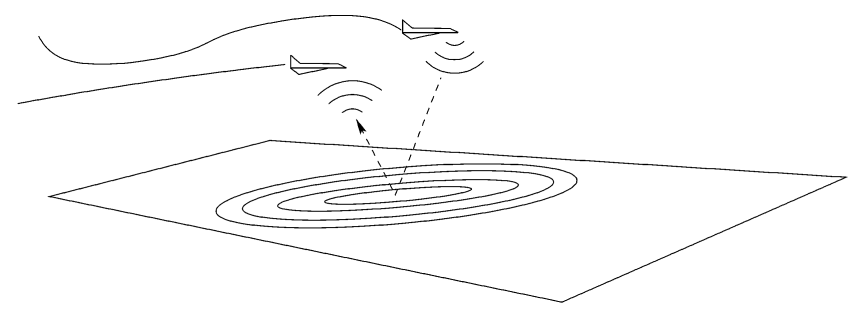

Fig. 2. Acquisition geometry for BISAR.

For SAR systems whose antennas are able to form a narrow beam, the image reconstruction algorithms are well known [5]-[12]. However, these algorithms are not useful for imaging systems using antennas having poor directivity where the antenna footprint is large.

In [13]-[17], reconstruction algorithms for monostatic SAR with poor antenna directivity traversing straight and arbitrary flight trajectories have been developed. To our knowledge, the acquisition geometry of BISAR studies for the case of poor antenna directivity are limited to isotropic antennas traversing certain flight trajectories (straight [18], [19] or circular [20], [21] flight trajectories) over flat topography.

In this paper, we focus on BISAR with poor antenna directivity and address the image reconstruction problem when transmitter and receiver are traversing arbitrary, but known, flight trajectories over a known, but not necessarily flat, topography.

In particular, we have used microlocal techniques to develop an approximate analytic image reconstruction method for BISAR. Microlocal techniques leads to inversion methods that have the desirable property that visible edges in the scene will appear in the reconstructed image at the correct location and orientation. They can also account for various factors such as the arbitrary flight trajectory, nonflat ground topography, antenna beam pattern, transmitted waveform and geometric spreading. Furthermore, microlocal techniques give rise to filtered-backprojection (FBP-) type inversion, which is a direct 
(noniterative) method and can be potentially implemented fast [16]. If an exact inversion is possible, the FBP-type inversion often reduces to the exact inversion formula.

We describe the steps of the algorithm for numerical implementation of the inversion method and analyze its computational complexity. We present the performance of the algorithm in numerical simulations using circular and distorted circular flight trajectories over flat topography. Numerical simulations support the theory and show that the edges are recovered at the correct location and orientation and at the correct strength.

The organization of the paper is as follows. In Section II, we introduce our forward model. In Section III, we present the FBP-type image formation method along with an example. In Section IV, we present the reconstruction algorithm and its computational complexity analysis and numerical simulations to illustrate our theoretical results and the performance of the reconstruction algorithm. Our conclude our discussion with Section V. Finally, we provide the stationary phase theorem in the Appendix.

\section{FORWARD MODEL}

Let $\boldsymbol{\gamma}_{T}(s), \boldsymbol{\gamma}_{R}(s) \in \mathbb{R}^{3}, s \in \mathbb{R}$, be the transmitter and receiver trajectories, respectively. We assume that the earth's surface is located at the position $\mathbf{x}=\left(x_{1}, x_{2}, \psi\left(x_{1}, x_{2}\right)\right)$, where $\psi: \mathbb{R}^{2} \rightarrow \mathbb{R}$, is a known smooth function, and scattering takes place in a thin region near the surface. Following [14], [15], and [22], under the single-scattering (Born) approximation, we model the received signal $d(s, t)$ as follows:

$$
\begin{aligned}
d(s, t) & \approx \mathcal{F}[T](s, t) \\
& :=\int \mathrm{e}^{-\mathrm{i} 2 \pi \omega\left(t-R_{T R}(s, \mathbf{x}) / c_{0}\right)} A_{T R}(\boldsymbol{x}, s, \omega) T(\boldsymbol{x}) d \omega d \boldsymbol{x}
\end{aligned}
$$

where $\boldsymbol{x}=\left(x_{1}, x_{2}\right), R_{T R}(s, \mathbf{x})=\left|\boldsymbol{\gamma}_{T}(s)-\mathbf{x}\right|+\left|\mathbf{x}-\boldsymbol{\gamma}_{R}(s)\right|$ is the total traveled distance, also known as the bistatic range [1], $T(\boldsymbol{x})$ denotes the surface reflectivity, $c_{0}$ denotes the speed of light and $\omega$ denotes the temporal frequency. $A_{T R}$ is a complex amplitude function that includes the transmitter and receiver antenna beam patterns, the transmitted waveform, geometrical spreading factors, etc. [14], [15], [22]. For example, for an isotropic transmitter transmitting a waveform $P(\omega)$ and isotropic receiver, $A_{T R}(\boldsymbol{x}, s, \omega)$ can be approximated by $A_{T R}(\boldsymbol{x}, s, \omega)=P(\omega)\left(4 \pi\left|\gamma_{T}(s)-\mathbf{x}\right|\left|\gamma_{R}(s)-\mathbf{x}\right|\right)^{-1}$. Here $t$ denotes the fast time and $s$, which is referred to as the slow time, parameterizes the trajectory.

Unless otherwise stated, the bold Roman, bold italic, and italic small letters will denote points in $\mathbb{R}^{3}, \mathbb{R}^{2}$, and $\mathbb{R}$, respectively, i.e., $\mathbf{x}=\left(\boldsymbol{x}, x_{3}\right) \in \mathbb{R}^{3}$, with $\boldsymbol{x} \in \mathbb{R}^{2}$, and $x_{3} \in \mathbb{R}$.

We assume that for some $m_{A_{T R}}, A_{T R}$ satisfies the symbol estimate [14], [15]

$$
\begin{aligned}
\sup _{(s, \boldsymbol{x}) \in K}\left|\partial_{\omega}^{\alpha} \partial_{s}^{\beta} \partial_{x_{1}}^{\rho_{1}} \partial_{x_{2}}^{\rho_{2}} A_{T R}(\boldsymbol{x}, s, \omega)\right| & \\
& \leq C_{0}\left(1+\omega^{2}\right)^{\left(m_{A}-|\alpha|\right) / 2}
\end{aligned}
$$

where $K$ is any compact subset of $\mathbb{R} \times \mathbb{R}^{2}$, and the constant $C_{0}$ depends on $K$ and integers $\alpha, \beta, \rho_{1}$, and $\rho_{2}$. This assumption is true when the antenna is broadband and the source waveform is a band-limited waveform.

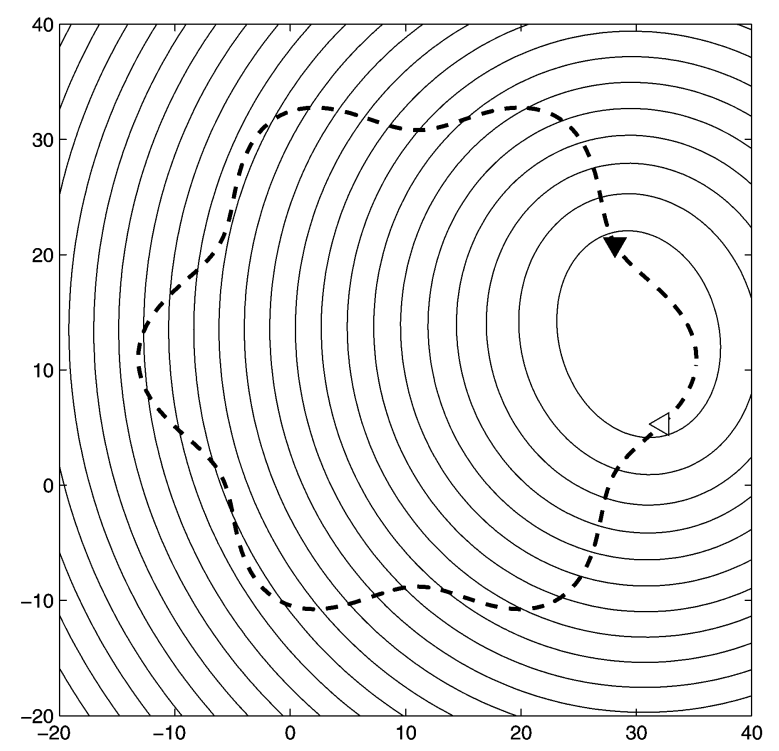

Fig. 3. Isorange contour for (dashed line) a distorted circular flight trajectory $\gamma_{T}(s)=\gamma_{R}(s+\pi / 4)=\gamma_{d}(s)$ over a flat topography where black and white triangles are the transmitter and receiver platforms, respectively [see Section IV for an explicit formula of $\left.\gamma_{d}(s)\right]$

In fact, under assumption (2), (1) defines $\mathcal{F}$ as a Fourier integral operator [23]-[25] whose leading order contribution comes from those points lying in the critical set of the phase, i.e., intersection of the illuminated surface and the ellipsoid $\mathcal{E}(s)=\{\mathbf{z}$ : $\left.R_{T R}(s, \mathbf{z})=c_{0} t\right\}$. Since $\mathcal{F}$ is a Fourier integral operator, an approximate inverse of $\mathcal{F}$ can be computed by a suitable backprojection onto these intersections. The curves formed by the intersection of the surface and $E_{s}$ are also referred to as iso-range contours [1] for the slow-time $s$. For flat topography $\psi(\boldsymbol{x})=0$, the iso-range contours are given by ellipses on the plane $x_{3}=0$ (see Fig. 3), which we will revisit in Section III-C.

The ideal image formation problem is to estimate $T$ from knowledge of $d(s, t)$ for some range of $s$ and $t$.

\section{IMAGE FORMATION}

In general, the strategy for estimating $T$ is to apply an imaging operator $\mathcal{K}$ to the data $\mathcal{F}[T]$. The image $\tilde{T}$ for the target can, thus, be written $\tilde{T}=\mathcal{K} \mathcal{F}[T]$. The operator $\mathcal{L}=\mathcal{K} \mathcal{F}$ contains the information about how the image $\tilde{T}$ is related to the actual target scene $T$. The kernel of $\mathcal{L}$ is called the point spread function.

Since $\mathcal{F}$ is a Fourier integral operator, an approximate inverse of $\mathcal{F}$ can be computed by another Fourier integral operator $\mathcal{K}$. Our strategy is to determine $\mathcal{K}$ so that the point spread function of $\mathcal{L}$ approximates the Dirac delta function. In this regard, we extend the monostatic SAR reconstruction techniques based on microlocal analysis [14], [15] to BISAR to determine $\mathcal{K}$.

The microlocal-analysis-based reconstruction method can be viewed as a generalized FBP-type reconstruction method where the data is first filtered and then backprojected. It is a direct consequence of microlocal analysis of the backprojection operator that the visible edges of the scene appear in the correct location and correct orientation in the image obtained by backprojection [14], [15]. 


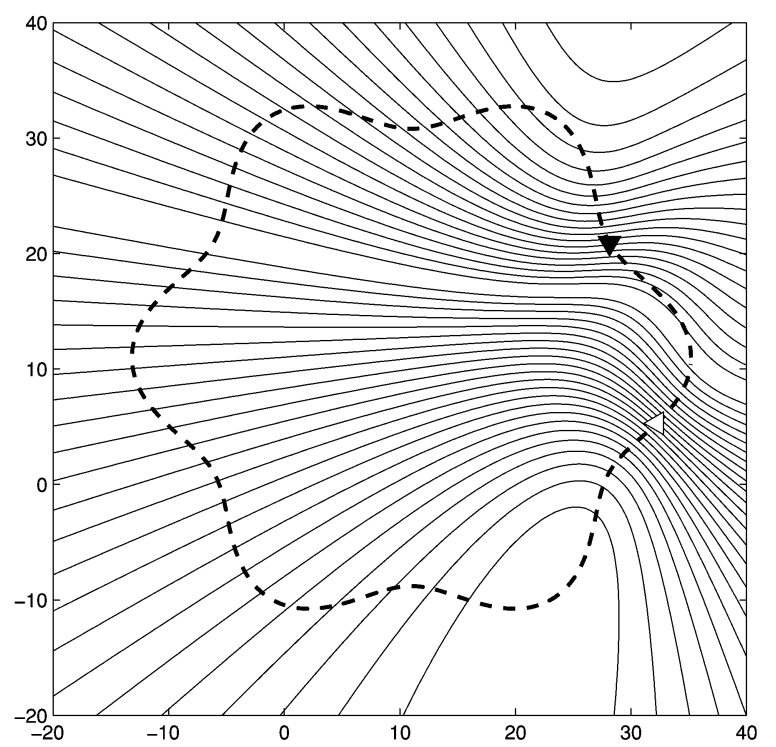

Fig. 4. Iso-doppler contour for (dashed line) a distorted circular flight trajectory $\boldsymbol{\gamma}_{T}(s)=\gamma_{R}(s+\pi / 4)=\boldsymbol{\gamma}_{d}(s)$ over a flat topography where black and white triangles are the transmitter and receiver platforms, respectively [see Section IV for an explicit formula of $\left.\gamma_{d}(s)\right]$.

\section{A. FBP Operator $\mathcal{K}$}

We use the following FBP operator $\mathcal{K}$ to form an image $\tilde{T}$ of the scene:

$$
\begin{aligned}
\tilde{T}(z):= & \mathcal{K}[d](\boldsymbol{z}) \\
:= & \int \mathrm{e}^{\mathrm{i} 2 \pi \omega\left(t-R_{T R}(s, \mathbf{z}) / c_{0}\right)} \\
& \times Q_{T R}(\boldsymbol{z}, s, \omega) d(s, t) d \omega d s d t \\
= & \int \mathrm{e}^{-\mathrm{i} 2 \pi \omega R_{T R}(s, \mathbf{z}) / c_{0}} \\
& \times Q_{T R}(\boldsymbol{z}, s, \omega) D(s, \omega) d \omega d s
\end{aligned}
$$

where $z=\left(z_{1}, z_{2}\right), \mathbf{z}=(z, \psi(z)), D(s, \omega)=$ $\int d(s, t) \exp (\mathrm{i} 2 \pi t \omega) d t$ is the Fourier transform of $d$ in fast-time, and $Q_{T R}$ is the filter to be determined below. We assume that for some $m_{Q}$ satisfies the symbol estimate

$$
\begin{aligned}
\sup _{(s, \boldsymbol{x}) \in K}\left|\partial_{\omega}^{\alpha} \partial_{s}^{\beta} \partial_{x_{1}}^{\rho_{1}} \partial_{x_{2}}^{\rho_{2}} Q_{T R}(z, s, \omega)\right| & \\
& \leq C_{0}\left(1+\omega^{2}\right)^{\left(m_{Q}-|\alpha|\right) / 2}
\end{aligned}
$$

where $K$ is any compact subset of $\mathbb{R} \times \mathbb{R}^{2}$, and the constant $C_{0}$ depends on $K, \alpha, \beta, \rho_{1}$, and $\rho_{2}$. Assumption (4) makes $\mathcal{K}$ a Fourier integral operator. In the next section, we will determine the filter $Q_{T R}$ and see that $Q_{T R}$ indeed satisfies the assumption (4).

Substituting (1) into (3) results in

$$
\tilde{T}(\boldsymbol{z})=\mathcal{K} \mathcal{F}[T](\boldsymbol{z})=\mathcal{L}[T](z)=\int L(\boldsymbol{z}, \boldsymbol{x}) T(\boldsymbol{x}) d \boldsymbol{x}
$$

where

$$
\begin{aligned}
L(\boldsymbol{z}, \boldsymbol{x})=\int \mathrm{e}^{\mathrm{i} 2 \pi \omega \phi(\boldsymbol{x}, \boldsymbol{z}, \boldsymbol{s}) / c_{0}} & \\
& \times Q_{T R}(\boldsymbol{z}, s, \omega) A_{T R}(\boldsymbol{x}, s, \omega) d \omega d s
\end{aligned}
$$

is the point spread function with $\phi(\boldsymbol{x}, \boldsymbol{z}, s)=R_{T R}(s, \mathbf{x})-$ $R_{T R}(s, \mathbf{z})$. We would like to make $L(\boldsymbol{z}, \boldsymbol{x})$ as close as possible to the Dirac delta function $\delta(\boldsymbol{x}-\boldsymbol{z})=\int \exp (\mathrm{i} 2 \pi(\boldsymbol{x}-\boldsymbol{z}) \cdot \boldsymbol{\xi}) d \boldsymbol{\xi}$.

Applying the stationary phase theorem (see Appendix) to the $\omega$ and $s$ integrations in (6), we see that the main contributions to $L(\boldsymbol{z}, \boldsymbol{x})$ come from those critical points of its phase at which $A_{T R}$ is nonzero. Thus, the critical points satisfy

$$
\begin{aligned}
\partial_{\omega}[\omega \phi(\boldsymbol{x}, \boldsymbol{z}, s)] & =0 \Longrightarrow R_{T R}(s, \mathbf{x})=R_{T R}(s, \mathbf{z}) \\
\partial_{s}[\omega \phi(\omega, \boldsymbol{z}, \boldsymbol{x}, s)] & =0 \Longrightarrow f_{T R}(s, \mathbf{x})=f_{T R}(s, \mathbf{z})
\end{aligned}
$$

where

$$
\begin{aligned}
f_{T R}(s, \mathbf{x})=\omega\left[\dot { \gamma } _ { T } ( s ) \cdot \left(\gamma_{T} \widehat{(s)}-\right.\right. & \mathbf{x}) \\
& \left.+\dot{\gamma}_{R}(s) \cdot\left(\gamma_{R} \widehat{(s)}-\mathbf{x}\right)\right]
\end{aligned}
$$

is the bistatic Doppler frequency [1] with $\dot{\boldsymbol{\gamma}}_{T, R}=\partial \boldsymbol{\gamma}_{T, R}(s) / \partial s$ being the partial derivative of the trajectories with respect to $s$ and $\widehat{\mathbf{x}}$ denoting the unit vector along $\mathbf{x}$. The contours that are formed by the intersection of the surface and $\left\{\mathbf{x}: f_{T R}(s, \mathbf{x})=\right.$ $C$ \} for some constant $C$ are referred to as the iso-doppler contours (see Fig. 4). Thus, the critical points $\mathbf{z}$ are those that have the same bistatic range and Doppler as $\mathbf{x}$. In other words, $\mathbf{z}$ is a point that belongs to the intersection of the iso-range and iso-doppler contours from $\mathbf{x}$. For the rest of the paper, we will assume that the flight trajectories and antenna beam patterns of the transmitter and receiver are focused to one side of their flight trajectories, so that the iso-range and iso-doppler curves intersect only at a single point. Thus, we assume that the only critical point of the intersection is $z=\boldsymbol{x}$.

For fixed $\boldsymbol{x}$ and $\boldsymbol{z}$, we write $\phi(\boldsymbol{z}, \boldsymbol{x}, s)$ as

$$
\phi(\boldsymbol{x}, \boldsymbol{z}, s)=(\boldsymbol{x}-\boldsymbol{z}) \cdot \boldsymbol{\Xi}(s, \boldsymbol{x}, \boldsymbol{z})
$$

where

$$
\boldsymbol{\Xi}(s, \boldsymbol{x}, \boldsymbol{z})=\int_{0}^{1} \nabla R_{T R}(s, \boldsymbol{z}+\lambda(\boldsymbol{x}-\boldsymbol{z})) d \lambda .
$$

Here, we use the identity

$$
h(\boldsymbol{x})-h(\boldsymbol{z})=(\boldsymbol{x}-\boldsymbol{z}) \cdot \int_{0}^{1} \nabla h(\boldsymbol{z}+\lambda(\boldsymbol{x}-\boldsymbol{z})) d \lambda
$$

with $h(\boldsymbol{x})=R_{T R}(s, \boldsymbol{x})$. For $\boldsymbol{x}=z$

$$
\boldsymbol{\Xi}(s, \boldsymbol{z}, \boldsymbol{z})=\left[\mathrm{J}\left(z_{1}, z_{2}\right)\right]^{T}\left[\left(\boldsymbol{\gamma}_{T} \widehat{(s)}-\mathbf{z}\right)+\left(\boldsymbol{\gamma}_{R} \widehat{(s)}-\mathbf{z}\right)\right]
$$


where

$$
\begin{aligned}
& \mathrm{J}\left(z_{1}, z_{2}\right)=\left[\partial \mathbf{z} / \partial z_{1} \quad \partial \mathbf{z} / \partial z_{2}\right] \\
& =\left[\begin{array}{cc}
1 & 0 \\
0 & 1 \\
\partial \psi / \partial z_{1} & \partial \psi / \partial z_{2}
\end{array}\right]
\end{aligned}
$$

and superscript $T$ denotes transposition. Note that the columns of $\mathrm{J}$ span the tangent plane of the surface at point $\mathbf{z}$. Thus, $\boldsymbol{\Xi}(s, \boldsymbol{z}, \boldsymbol{z})$ is given by the projection of $\left[\left(\gamma_{T} \widehat{(s)}-\mathbf{z}\right)+\left(\gamma_{R} \widehat{(s)}-\mathbf{z}\right)\right]$ onto the tangent plane at $\mathbf{z}$.

Substituting (10) into (6), we obtain the point spread function becomes

$$
\begin{aligned}
& L(\boldsymbol{z}, \boldsymbol{x})=\int \mathrm{e}^{\mathrm{i} 2 \pi(\boldsymbol{x}-\boldsymbol{z}) \cdot \omega \boldsymbol{\Xi}(s, \boldsymbol{x}, \boldsymbol{z}) / c_{0}} \\
& \times Q_{T R}(z, s, \omega) A_{T R}(\boldsymbol{x}, s, \omega) d \omega d s .
\end{aligned}
$$

Under the assumptions (2) and (4), $\mathcal{L}$ becomes a pseudo-differential operator [23]-[25], which implies that the location and the orientation of the visible edges will be correctly reconstructed.

\section{B. Filter $Q_{T R}$}

In order to reconstruct the edges with the correct strength (i.e., magnitude of the jump) and order (i.e., not smoothed), we use the filter $Q_{T R}$ obtained from the following argument.

For fixed $\boldsymbol{x}$ and $z$, we make the change of variables

$$
(s, \omega) \rightarrow \boldsymbol{\xi}=\frac{\omega}{c_{0}} \mathbf{\Xi}(s, \boldsymbol{x}, \boldsymbol{z})
$$

in the integral of (15), and obtain

$$
\begin{aligned}
L(\boldsymbol{z}, \boldsymbol{x})=\int \mathrm{e}^{\mathrm{i} 2 \pi(\boldsymbol{x}-\boldsymbol{z}) \cdot \boldsymbol{\xi}} & \\
& \times Q_{T R}(\boldsymbol{z}, \boldsymbol{\xi}) A_{T R}(\boldsymbol{x}, \boldsymbol{\xi}) \eta(\boldsymbol{x}, \boldsymbol{z}, \boldsymbol{\xi}) d \boldsymbol{\xi}
\end{aligned}
$$

where $Q_{T R}(\boldsymbol{z}, \boldsymbol{\xi})=Q_{T R}(\boldsymbol{z}, s(\boldsymbol{\xi}), \omega(\boldsymbol{\xi}))$, etc., and

$$
\eta(\boldsymbol{x}, \boldsymbol{z}, \boldsymbol{\xi})=\left|\frac{\partial(s, \omega)}{\partial \boldsymbol{\xi}}\right|
$$

is the determinant of the Jacobian that comes from the change of variables (16).

Substituting (17) into (5) gives

$$
\begin{aligned}
\tilde{T}(\boldsymbol{z}) \approx \int \mathrm{e}^{\mathrm{i} 2 \pi(\boldsymbol{x}-\boldsymbol{z}) \cdot \boldsymbol{\xi}} \eta(\boldsymbol{x}, \boldsymbol{z}, \boldsymbol{\xi}) T(\boldsymbol{x}) & \\
& \times Q_{T R}(\boldsymbol{z}, \boldsymbol{\xi}) A_{T R}(\boldsymbol{x}, \boldsymbol{\xi}) d \boldsymbol{x} d \boldsymbol{\xi} .
\end{aligned}
$$

A stationary phase analysis of (19) in the variables $\boldsymbol{\xi}$ and $\boldsymbol{x}$ can be carried out as follows: First we make a change of variables $\xi=\lambda \xi^{\prime}$ to obtain a large parameter $\lambda$. We also write the amplitude $f=Q_{T R} A_{T R} \eta T$ in terms of its Fourier transform

$$
f(\boldsymbol{x})=\int e^{-2 \pi i \boldsymbol{x} \cdot \boldsymbol{\zeta}} \widehat{f}(\boldsymbol{\zeta}) d \boldsymbol{\zeta}
$$

and again make the change of variables $\zeta=\lambda \zeta^{\prime}$ to introduce the same large parameter $\lambda$. The critical conditions are $x=z$ and $\xi^{\prime}=\zeta^{\prime}$. Thus, we see that the leading-order contribution to (19) comes from $x=z$.
At this point, we see that the desired choice of the filter $Q_{T R}$ is

$$
Q_{T R}(z, s, \omega)=\chi_{\Omega_{\boldsymbol{z}}}(\boldsymbol{\xi}(s, \omega)) \frac{\overline{A_{T R}(\boldsymbol{z}, s, \omega)}}{\left|A_{T R}(z, s, \omega)\right|^{2}} \frac{1}{\eta(z, z, \xi)}
$$

where

$$
\Omega_{z}=\left\{\boldsymbol{\xi}=\frac{\omega}{c_{0}} \mathbf{\Xi}(s, z, z) \mid A_{T R}(z, s, \omega) \neq 0\right\}
$$

and $\chi_{\Omega_{z}}$ is a smooth cut-off function equal to one in the interior of $\Omega_{z}$ and zero in the exterior of $\Omega_{z}$. We refer to (22) as the data collection manifold at $z$; it is this set that determines many of the properties of the image. We note that since $A_{T R}$ satisfies the symbol estimate (2), by (21), $Q_{T R}$ also satisfies the symbol estimate (4) [23]-[25].

With the choice (21), the leading-order contribution to (19) becomes simply $T(z)$. However, it is more illuminating to use (21) in (19) and use the fact that the leading-order contribution is at $z=x$ to write

$$
\begin{aligned}
\tilde{T}(\boldsymbol{z}) & =\mathcal{K} \mathcal{F}[T](\boldsymbol{z}) \\
& \approx \iint_{\Omega_{\boldsymbol{z}}} \mathrm{e}^{\mathrm{i} 2 \pi(\boldsymbol{x}-\boldsymbol{z}) \cdot \boldsymbol{\xi}_{\chi_{\Omega_{z}}}(\boldsymbol{\xi}) T(\boldsymbol{x}) d \boldsymbol{\xi} d \boldsymbol{x} .}
\end{aligned}
$$

Equation (23) shows that the image $\tilde{T}$ is a band-limited version of $T$ whose frequency content, using (16), is determined by the union $\bigcup_{z} \Omega_{z}$. The data collection manifold $\Omega_{z}$ describes the frequency content and, thus, the resolution of the reconstructed image $\tilde{T}$ at $z$. The bigger the data collection manifold, the better the resolution of the reconstructed image. Microlocal analysis of (23) tells us that an edge at $z$ is visible if the direction $n_{z}$ normal to the edge is contained in $\Omega_{z}$ [14], [15], [26]. Furthermore, the frequency contribution of $\omega \Xi(s, z, z)$ to a visible edge at $z$ is given by $\cup_{\omega}\|\omega \boldsymbol{\Xi}(s, \boldsymbol{z}, \boldsymbol{z})\|$, where $\|\cdot\|$ denotes the Euclidean length. Thus, by (23), one can only reconstruct the visible edges of $T$ in the aforementioned sense. With the choice of the filter in (21), edges are also recovered at the right strength and order.

The $\overline{A_{T R}} /\left|A_{T R}\right|^{2}$ part of the filter $Q_{T R}$ includes a number of operations such as matched filtering, compensation of geometrical spreading factors, etc.

Connections with the tomography literature can be seen from closer examination of the Jacobian determinant appearing in (21). In particular, let $\boldsymbol{\Xi}(\boldsymbol{z}, \boldsymbol{z}, s)=\left(\Xi_{1}, \Xi_{2}\right)$ and $\partial \boldsymbol{\Xi}(z, z, s) / \partial s=\left(\dot{\Xi}_{1}, \dot{\Xi}_{2}\right)$. Then, by (13), (16), and (18), we have

$$
\begin{aligned}
\frac{1}{\eta(z, z, \boldsymbol{\xi})} & =\left|\frac{\partial \boldsymbol{\xi}}{\partial(s, \omega)}\right| \\
& =\frac{|\omega|}{c_{0}^{2}}\left|\Xi_{1} \dot{\Xi}_{2}-\Xi_{2} \dot{\Xi}_{1}\right| .
\end{aligned}
$$

In the tomography literature [27], $|\omega|$ in (24) is referred to as the Ram-Lak or the ramp filter. Its linear growth corresponds to a derivative; without it, the edges appear smoothed. We see that, in general, $Q_{T R}$ is a combination of the matched filter and ramp filter together with an additional weighting factor due to 
geometric spreading and a local scaling factor $\left|\Xi_{1} \dot{\Xi}_{2}-\Xi_{2} \dot{\Xi}_{1}\right|$. In most radar systems, however, issues associated with the absolute value $|\omega|$ do not arise, because the received signal is demodulated in such a way that $\omega$ for the "analytic signal" can be assumed to be positive.

\section{Example: Isotropic Antenna and Flat Topography}

Let $A_{T R}=1$, which corresponds to an isotropic transmit antenna radiating a delta-like impulse, an isotropic receive antenna and compensation of geometric spreading factors in the data. Then, using the definition of Dirac delta function and its homogeneity property, we have

$d(s, t) \approx c_{0} \int \delta\left(c_{0} t-\left(\left|\boldsymbol{\gamma}_{T}(s)-\mathbf{x}\right|+\left|\mathbf{x}-\gamma_{R}(s)\right|\right)\right) T(\boldsymbol{x})$.

The expression inside the Dirac delta defines an ellipsoid. Thus, (25) gives us the integral of $T$ along the curves obtained by the intersection of $(\boldsymbol{x}, \psi(\boldsymbol{x}))$ and the ellipsoid $\mathcal{E}(s)=\left\{\mathbf{x} \in \mathbb{R}^{3}\right.$ : $\left.\left|\boldsymbol{\gamma}_{T}(s)-\mathbf{x}\right|+\left|\mathbf{x}-\boldsymbol{\gamma}_{R}(s)\right|=c_{0} t\right\}$.

Let the topography be flat, i.e., $\psi(x)=0$. Then the equation of the ellipse formed by the intersection of the plane $x_{3}=0$ and the ellipsoid $\mathcal{E}(s)$ is

$$
a x_{1}^{2}+b x_{1} x_{2}+c x_{2}^{2}+2 d x_{1}+2 f x_{2}+g=0
$$

where $a, b, c, d, f$, and $g$ are defined in the first equation shown at the bottom of the page. For notational brevity, we will omit the $s$ dependence. Let $\left(c_{1}, c_{2}\right)=\left(c_{1}(s), c_{2}(s)\right)$ denote the center and $M=M(s), m=m(s)$ denote the major and minor axis, respectively, and $\varphi=\varphi(s)$ denote the angle of rotation of the ellipse. Then $M, m$, and $\varphi$ are given by the second equation shown at the bottom of the page. Let

$$
\begin{aligned}
& p_{1}(\theta)=c_{1}+m \cos \varphi \cos \theta+M \sin \varphi \sin \theta \\
& p_{2}(\theta)=c_{2}+M \cos \varphi \sin \theta-m \sin \varphi \cos \theta
\end{aligned}
$$

Thus, in polar coordinates, (25) becomes

$$
d(s, t) \approx c_{0}^{2} \int_{0}^{2 \pi} T\left(p_{1}(\theta), p_{2}(\theta)\right) \sqrt{M m} d \theta
$$

providing an efficient way to implement (25).

Under the flat topography assumption, by (21), the backprojection filter is given by

$$
\begin{aligned}
Q_{T R}(z, s, \omega) & =\chi_{\Omega_{z}}(\boldsymbol{\xi}(s, \omega))\left|\frac{\partial \xi}{\partial(\omega, s)}\right| \\
& =\chi_{\Omega_{z}}(\boldsymbol{\xi}(s, \omega)) \frac{|\omega|}{c_{0}^{2}} \tilde{Q}_{T R}(z, s)
\end{aligned}
$$

$$
\begin{aligned}
& a=4\left(\gamma_{T 1}^{2}-2 \gamma_{R 1} \gamma_{T 1}+\gamma_{R 1}^{2}-\left(c_{0} t\right)^{2}\right) \\
& b=4\left(\gamma_{R 2}-\gamma_{T 2}\right)\left(\gamma_{R 1}-\gamma_{T 1}\right) \\
& c=4\left(\gamma_{R 2}^{2}-2 \gamma_{R 2} \gamma_{T 2}+\gamma_{T 2}^{2}-\left(c_{0} t\right)^{2}\right) \\
& d=2\left[\sum_{i=1}^{3}\left(\gamma_{T i}^{2}-\gamma_{R i}^{2}\right)\left(\gamma_{R 1}-\gamma_{T 1}\right)+\left(c_{0} t\right)^{2}\left(\gamma_{R 1}+\gamma_{T 1}\right)\right] \\
& f=2\left[\sum_{i=1}^{3}\left(\gamma_{T i}^{2}-\gamma_{R i}^{2}\right)\left(\gamma_{R 2}-\gamma_{T 2}\right)+\left(c_{0} t\right)^{2}\left(\gamma_{R 2}+\gamma_{T 2}\right)\right] \\
& g=\left[\sum_{i=1,2,3}\left(\gamma_{R i}^{4}+\gamma_{T i}^{4}\right)-2\left(c_{0} t\right)^{2} \sum_{i=1}^{3}\left(\gamma_{R i}^{2}+\gamma_{T i}^{2}\right)\right] \\
& \left.+\sum_{i, j=1,2,3}^{2} \gamma_{T i}^{2} \gamma_{T j}^{2}+\gamma_{R i}^{2} \gamma_{R j}^{2}-\gamma_{T i}^{2} \gamma_{R j}^{2}+\left(c_{0} t\right)^{4}\right]
\end{aligned}
$$

$$
\begin{aligned}
\left(c_{1}, c_{2}\right) & =(c d-b f, a f-b d) /\left(b^{2}-a c\right) \\
M & =\max _{ \pm} \sqrt{\frac{2\left(a f^{2}+c d^{2}+g b^{2}-2 b d f-a c g\right)}{\left(b^{2}-a c\right)\left[(c \pm a) \sqrt{1+\frac{4 b^{2}}{(a-c)^{2}}}-(c+a)\right]}} \\
m & =\min _{ \pm} \sqrt{\frac{2\left(a f^{2}+c d^{2}+g b^{2}-2 b d f-a c g\right)}{\left(b^{2}-a c\right)\left[(c \pm a) \sqrt{1+\frac{4 b^{2}}{(a-c)^{2}}}-(c+a)\right]}} \\
\varphi & =\frac{1}{2} \cot ^{-1}\left(\frac{c-a}{2 b}\right)
\end{aligned}
$$


where the data collection manifold is

$$
\begin{aligned}
\Omega_{\boldsymbol{z}}=\{\omega \boldsymbol{\Xi}(s, \boldsymbol{z}, \boldsymbol{z})= & \omega \mathrm{J}^{T} \\
& \left.\times\left[\left(\boldsymbol{\gamma}_{T} \widehat{(s)}-\mathbf{z}\right)+\left(\boldsymbol{\gamma}_{R} \widehat{(s)}-\mathbf{z}\right)\right]\right\}
\end{aligned}
$$

with

$$
\mathrm{J}=\left[\begin{array}{ll}
1 & 0 \\
0 & 1 \\
0 & 0
\end{array}\right]
$$

Here

$$
\begin{aligned}
\tilde{Q}_{T R}(z, s) & \\
= & \mid Q_{T 12}+Q_{R 12}+Q_{T R 12}+Q_{R T 12} \\
& -Q_{T R 123}\left[Q_{R 123}-Q_{T 123}\right] \mid
\end{aligned}
$$

and

$$
\begin{aligned}
& Q_{T 12} \\
& =\frac{\left(\gamma_{T 1}-z_{1}\right) \dot{\gamma}_{T 2}-\left(\gamma_{T 2}-z_{2}\right) \dot{\gamma}_{T 1}}{\left|\boldsymbol{\gamma}_{T}-\mathbf{z}\right|^{2}}
\end{aligned}
$$

$Q_{R 12}$

$$
=\frac{\left(\gamma_{R 1}-z_{1}\right) \dot{\gamma}_{R 2}-\left(\gamma_{R 2}-z_{2}\right) \dot{\gamma}_{R 1}}{\left|\gamma_{R}-\mathbf{z}\right|^{2}}
$$

$Q_{T R 12}$

$$
=\frac{\left(\gamma_{R 1}-z_{1}\right) \dot{\gamma}_{T 2}-\left(\gamma_{R 2}-z_{2}\right) \dot{\gamma}_{T 1}}{\left|\gamma_{T}-\mathbf{z}\right|\left|\gamma_{R}-\mathbf{z}\right|}
$$

$Q_{R T 12}$

$$
=\frac{\left(\gamma_{T 1}-z_{1}\right) \dot{\gamma}_{R 2}-\left(\gamma_{T 2}-z_{2}\right) \dot{\gamma}_{R 1}}{\left|\gamma_{T}-\mathbf{z}\right|\left|\gamma_{R}-\mathbf{z}\right|}
$$

$Q_{T R 123}$

$$
=\frac{\left[\left(\gamma_{R 2}-z_{2}\right)\left(\gamma_{T 1}-z_{1}\right)-\left(\gamma_{R 1}-z_{1}\right)\left(\gamma_{T 2}-z_{2}\right)\right]}{\left|\gamma_{T}-\mathbf{z}\right|\left|\gamma_{R}-\mathbf{z}\right|}
$$

$Q_{R 123}$

$$
=\frac{\left(\gamma_{R 1}-z_{1}\right) \dot{\gamma}_{R 1}+\left(\gamma_{R 2}-z_{2}\right) \dot{\gamma}_{R 2}+\dot{\gamma}_{R 3} \gamma_{R 3}}{\left|\gamma_{R}-\mathbf{z}\right|^{2}}
$$

$Q_{T 123}$

$$
=\frac{\left(\gamma_{T 1}-z_{1}\right) \dot{\gamma}_{T 1}+\left(\gamma_{T 2}-z_{2}\right) \dot{\gamma}_{T 2}+\dot{\gamma}_{T 3} \gamma_{T 3}}{\left|\gamma_{T}-\mathbf{z}\right|^{2}} .
$$

1) Monostatic SAR: In monostatic SAR, $\boldsymbol{\gamma}_{T}=\boldsymbol{\gamma}_{R}=\boldsymbol{\gamma}$ and

$$
\begin{aligned}
Q_{T 12} & =Q_{R 12}=Q_{T R 12}=Q_{R T 12} \\
Q_{R 123} & =Q_{T 123}
\end{aligned}
$$

Thus, $Q_{T R}$ reduces to the filter $Q$ of the monostatic SAR [15]

$$
\begin{array}{r}
Q(z, s)=\chi_{\Omega_{\boldsymbol{z}}}(\boldsymbol{\xi}(s, \omega)) \\
\times \frac{4|\omega|}{c_{0}^{2}} \frac{\left(\gamma_{1}-z_{1}\right) \dot{\gamma}_{2}-\left(\gamma_{2}-z_{2}\right) \dot{\gamma}_{1}}{|\gamma-\mathbf{z}|^{2}} .
\end{array}
$$

Here, by (13) and (22), the data collection manifold is given by $\Omega_{\boldsymbol{z}}(\boldsymbol{\xi}(s, \omega))=\left\{\omega \boldsymbol{\Xi}_{\text {mono }}(s, \boldsymbol{z})\right\}$, where $\boldsymbol{\Xi}_{\text {mono }}(s, \boldsymbol{z})=$ $2 \mathrm{~J}^{T}(\boldsymbol{\gamma}(s)-\mathbf{z})$.

2) Static Transmitter: Let the transmitter be static, $\boldsymbol{\gamma}_{T}(s)=$ $\gamma_{T} \in \mathbb{R}^{3}$. This is the case for a passive surveillance scenario, when there is a remote high-powered transmitter on the ground or space, or a radio or television station and the receiver is

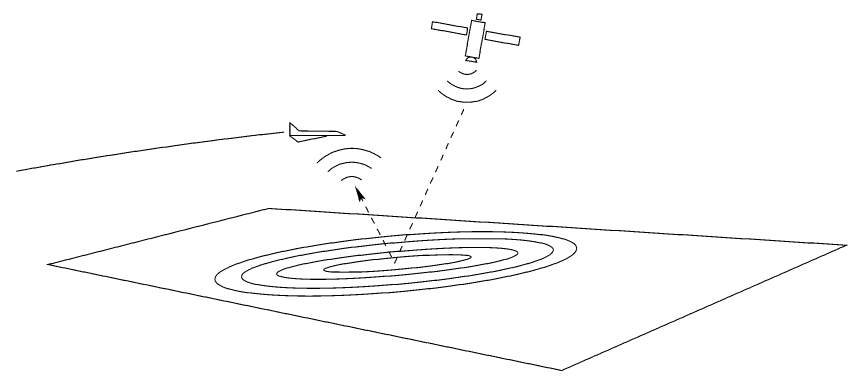

Fig. 5. Acquisition geometry for static transmitter.

mounted on an airborne platform flying over an area of interest, (see Fig. 5). Then

$$
Q_{T 12}=Q_{T R 12}=Q_{T 123}=0
$$

and, hence

$$
\begin{aligned}
\tilde{Q}_{T R}(z, s) & \\
= & \frac{\dot{\boldsymbol{\gamma}}_{R}(s)}{\left|\boldsymbol{\gamma}_{R}(s)-\mathbf{z}\right|} \cdot\left(\left[\left(\boldsymbol{\gamma}_{R} \widehat{(s)}-\mathbf{z}\right)^{\perp}+\left(\widehat{\boldsymbol{\gamma}_{T}-\mathbf{z}}\right)^{\perp}\right]\right. \\
& +\left[\left(\widehat{\left.\boldsymbol{\gamma}_{T}-\mathbf{z}\right)} \cdot\left(\boldsymbol{\gamma}_{R} \widehat{(s)}-\mathbf{z}\right)^{\perp}\right]\left(\boldsymbol{\gamma}_{R} \widehat{(s)}-\mathbf{z}\right)\right)
\end{aligned}
$$

where $\hat{\mathbf{x}}^{\perp}:=\left(-x_{2}, x_{1}, 0\right) /|\mathbf{x}|$. The data collection manifold is given by $\Omega_{\boldsymbol{z}}(\boldsymbol{\xi}(s, \omega))=\left\{\omega \boldsymbol{\Xi}_{\text {static }}(s, \boldsymbol{z})\right\}$, where $\boldsymbol{\Xi}_{\text {static }}(s, \boldsymbol{z})=\mathrm{J}^{T}\left[\left(\boldsymbol{\gamma}_{T}-\mathbf{z}\right)+\left(\boldsymbol{\gamma}_{R} \widehat{(s)}-\mathbf{z}\right)\right]$.

By reciprocity, (37) can be modified to obtain the filter for static receiver and mobile transmitter by interchanging $T$ and $R$.

\section{COMPUTATIONAL COMPLEXITy ANALysis AND NUMERICAL SIMULATIONS}

In this section, we will first present our numerical implementation of the FBP method, analyze its computational complexity and next present numerical simulations.

\section{A. Reconstruction Algorithm and Computational Complexity}

Substituting (30) into (3), we write the corresponding reconstruction formula for (25) as

$$
\begin{aligned}
\tilde{T}(\boldsymbol{z})=\int \tilde{Q}_{T R}(\boldsymbol{z}, s) & \\
\times & {\left[\int \mathrm{e}^{-\mathrm{i} 2 \pi \frac{\omega}{c_{0}} R_{T R}(s, \boldsymbol{z})} \frac{|\omega|}{c_{0}^{2}} D(s, \omega) d \omega\right] d s }
\end{aligned}
$$

where

$$
D(s, \omega)=\int \mathrm{e}^{\mathrm{i} 2 \pi t \omega} d(s, t) d t
$$

is the Fourier transform of $d(s, t)$ with respect to the fast-time.

We implemented the reconstruction formula (38) in four steps. Assuming there are $\mathcal{O}(N)$ samples in both the fast-time and slow-time variables and the image is of size $\mathcal{O}(N \times N)$, the steps of the reconstruction and their corresponding computational complexity are as follows.

1) Computing the Fourier Transform in Fast Time: For each $s$, (39) can be computed using the fast Fourier transform 

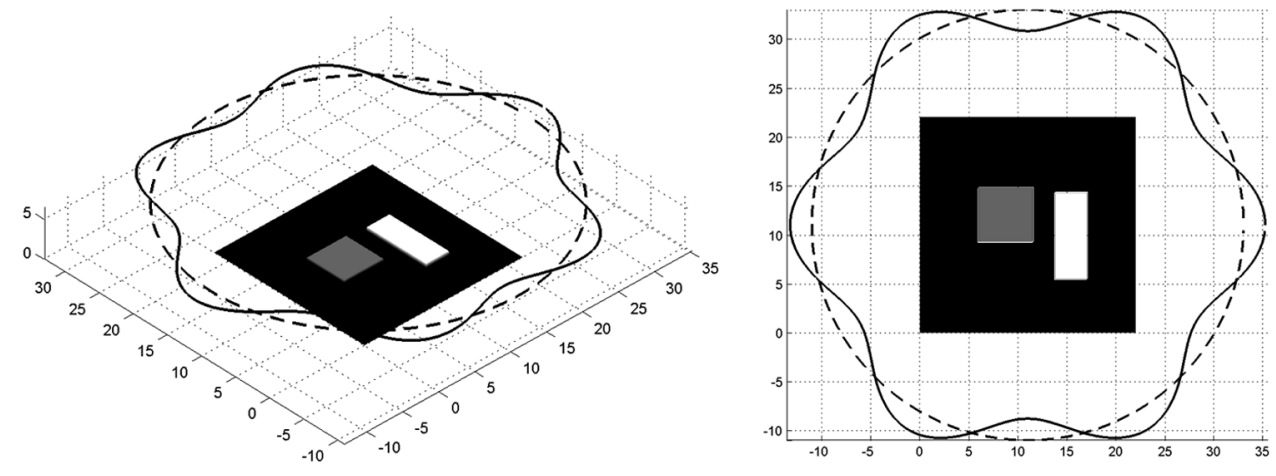

Fig. 6. (Left) 3-D and (right) 2-D views of (dashed line) circular and (solid line) distorted circular flight trajectories. The axes labels are in kms.

(FFT) in $\mathcal{O}(N \log N)$ computations. Thus, for all $s$, the computation complexity of this step is $\mathcal{O}\left(N^{2} \log N\right)$.

2) Ramp Filtering: Let

$$
\tilde{D}(s, \omega)=|\omega| D(s, \omega) .
$$

For each $s,(40)$ can be computed in $\mathcal{O}(N)$ number of computations. Thus, for all $s$, the computation complexity of this step is $\mathcal{O}\left(N^{2}\right)$.

3) Backprojection Operation: Let

$$
\tilde{T}_{s}(\boldsymbol{z})=\int \mathrm{e}^{-\mathrm{i} 2 \pi \frac{\omega}{c_{0}} R_{T R}(\boldsymbol{s}, \boldsymbol{z})} \tilde{D}(s, \omega) d \omega .
$$

For each $s$, (41) can be computed using FFT in $\mathcal{O}(N \log N)$ computations. Thus, for all $s$, the computational complexity of this step is $\mathcal{O}\left(N^{2} \log N\right)$. At this stage, the edges are positioned correctly in the image. In order to get the strengths correct, we need the following step.

4) Image Formation: Finally, we form the image $\tilde{T}$ by

$$
\tilde{T}(\boldsymbol{z})=\int \tilde{Q}(\boldsymbol{z}, s) \tilde{T}_{s}(\boldsymbol{z}) d s .
$$

The computational complexity for this step is $\mathcal{O}\left(N^{3}\right)$.

\section{B. Numerical Results}

We performed five numerical experiments: 1) monostatic SAR with circular transmitter and receiver flight trajectories, 2) bistatic SAR with circular transmitter and receiver flight trajectories, 3) bistatic SAR with a static transmitter and circular receiver flight trajectory, 4) bistatic SAR with a static transmitter and linear receiver flight trajectory, and 5) bistatic SAR with distorted circular transmitter and receiver flight trajectories.

Continuing with the example of Section III-C, in our numerical simulations, we considered a square target of size $5.5 \mathrm{~km}$ and a rectangular target of size $3.3 \times 8.8 \mathrm{~km}$ located in a scene of size $[0,22] \times[0,22] \mathrm{km}^{2}$ with their centers located at $(8.8,12) \mathrm{km}$ and $(15.4,10) \mathrm{km}$ (see Fig. 6). We discretize the scene with $128 \times 128$ pixels, where $(0,0,0) \mathrm{km}$ and $(22,22,0) \mathrm{km}$ correspond to the pixels $(1,1)$ and $(128,128)$, respectively (see Fig. 7). We used a discrete version of (29) to generate our simulation data. The parameters we used correspond roughly to a system bandwidth of $.873 \mathrm{MHz}$. In

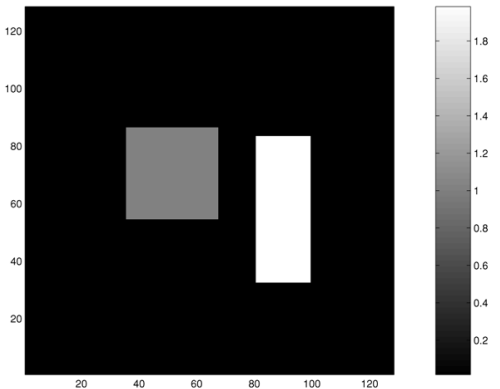

Fig. 7. Scene used in numerical simulations. The axes are labeled according to pixel number; this convention is also used for all the reconstructions below.

these experiments, we considered a circular flight trajectory $\boldsymbol{\gamma}(s)=(11+22 \cos s, 11+22 \sin s, 6.5) \mathrm{km}$, and a linear flight trajectory $\gamma_{l}(s)=(0,10 s / \pi, 6.5) \mathrm{km}$, uniformly sampled for $s \in[0,2 \pi]$ at 512 points.

For comparison purposes, we also reconstructed images using backprojection (BP) [18], [21] only, where we set $Q=1$.

For monostatic SAR (experiment 1), we set $\boldsymbol{\gamma}_{T}(s)=$ $\boldsymbol{\gamma}_{R}(s)=\gamma(s)$. The projection data and reconstructed images using BP and FBP are presented in Fig. 8.

For experiment 2 (BISAR with moving transmitter and receiver), both the transmitter and receiver move along the same path: we set the transmitter trajectory to $\boldsymbol{\gamma}_{T}(s)=\gamma(s)$ and the receiver trajectory to be $\boldsymbol{\gamma}_{R}(s)=\gamma(s+\pi / 4)$. The projection data and reconstructed images are presented in Fig. 9. For experiment 3 (BISAR with static transmitter), we fix the transmitter at the location $\boldsymbol{\gamma}_{T}=(0,0,6.5) \mathrm{km}$ and choose the receiver trajectory to be $\boldsymbol{\gamma}_{R}(s)=\boldsymbol{\gamma}(s)$. The projection data and reconstructed images are presented in Fig. 10. For experiment 4 (BISAR with static transmitter and linear receiver trajectory), we put the transmitter at $\boldsymbol{\gamma}_{T}=(0,0,6.5) \mathrm{km}$ and took the linear receiver trajectory to be $\boldsymbol{\gamma}_{R}(s)=\boldsymbol{\gamma}_{l}(s), s \in[0,2 \pi]$. The flight path and reconstruction are shown in Fig. 11. Finally, for experiment 5 (BISAR with perturbed circular trajectories), we used the trajectory $\gamma_{d}(s)=(11+22(\cos s+.1 \cos s \cos (6 s)), 11+$ $22(\sin s+.1 \sin s \cos (6 s)), 6.5) \mathrm{km}$ and took the transmitter and receiver flight trajectories to be $\boldsymbol{\gamma}_{T}(s)=\boldsymbol{\gamma}_{R}(s+\pi / 4)=$ $\gamma_{d}(s)$ (see Fig. 6). Again, we took 512 points, uniformly sampled in $s \in[0,2 \pi]$. The reconstructed images are presented in Fig. 12. All reconstructions are done with parameters that correspond roughly to a system bandwidth of $.873 \mathrm{MHz}$. 

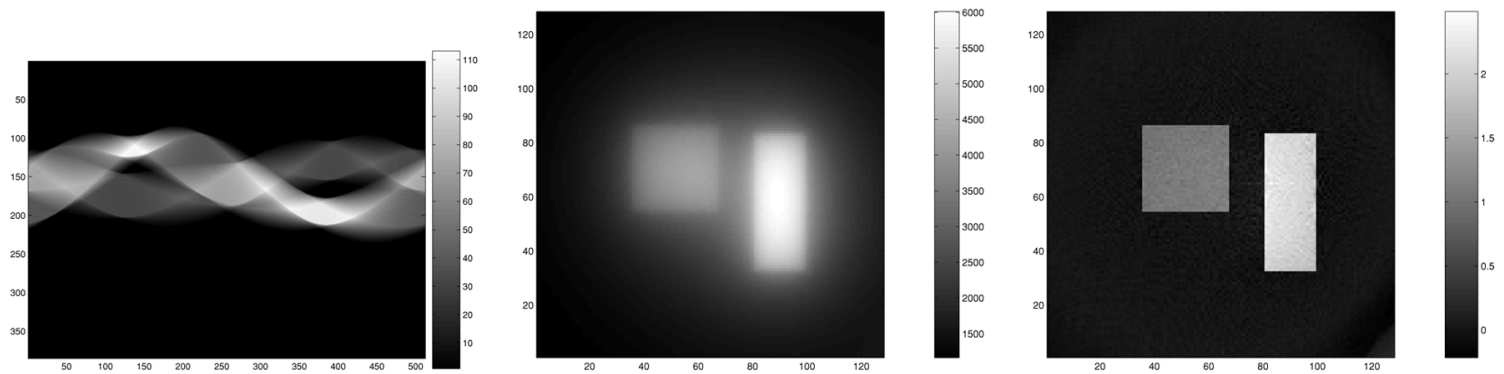

Fig. 8. (Left) Projection data for monostatic SAR with circular flight trajectory and reconstructed images obtained by (middle) backprojection and (right) FBP Both methods reconstruct the visible edges of the scene at the correct location and orientation. However, the edges in the backprojected image are smoothed; this is corrected in the FBP image, as predicted by the theory.
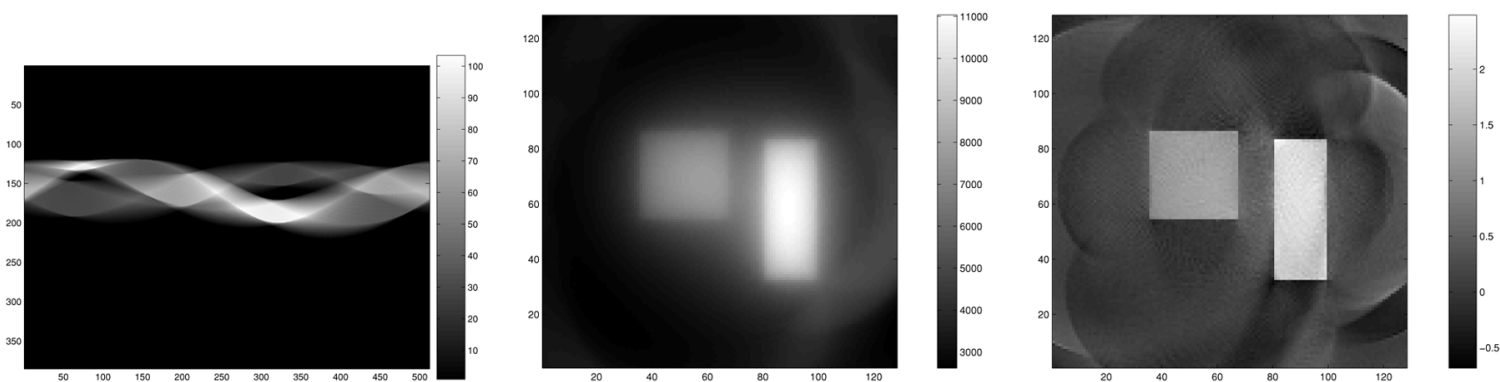

Fig. 9. (Left) Projection data for BISAR with circular transmitter and receiver trajectories, with reconstructed images obtained by (middle) backprojection and (right) FBP. Both methods reconstruct the visible edges of the scene at the correct location and orientation. However, the edges in the backprojected image are smoothed; this is corrected in the FBP image, as predicted by the theory.
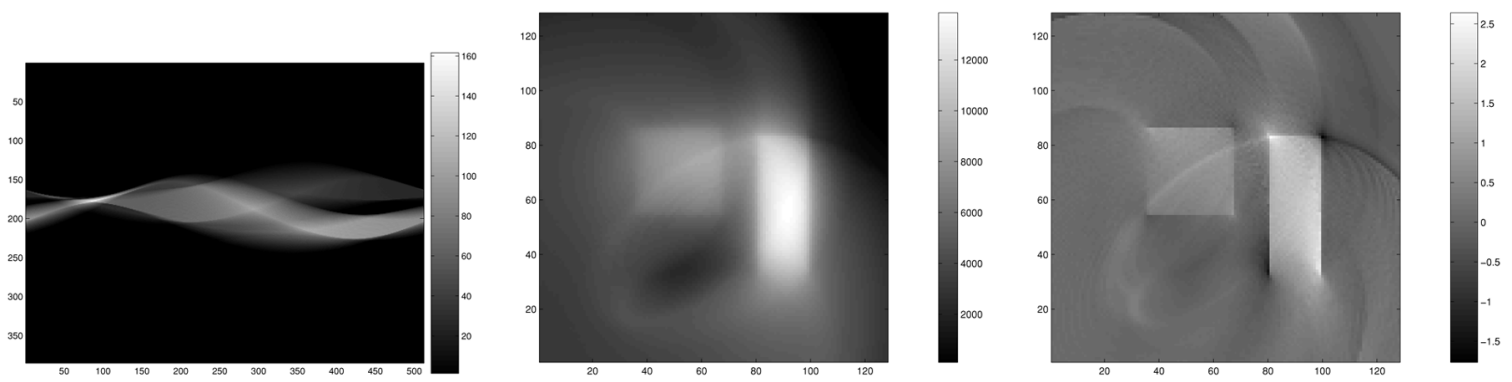

Fig. 10. (Left) Projection data for BISAR with a static transmitter and circular receiver trajectory, and reconstructed images obtained by (middle) backprojection and (right) FBP. Both methods reconstruct the visible edges of the scene at the correct location and orientation, but FBP provides sharper edges than BP method. These images are of lower quality than those of Fig. 9 because $\Omega_{z}$ is smaller.

Figs. 11 and 13 contain diagrams that show how the set (22) predicts what appears in the image. In particular, which edges are visible is determined by (22) and (13). For flat topography and a circular trajectory, both in monostatic SAR and in BISAR with a mobile transmitter, for each $z, \Omega_{z}$ contains all directions in $\mathbb{R}^{2}$. Since we are able to reconstruct edges that are perpendicular to the directions in $\Omega_{z}$, all the edges of the scene are visible. Fig. 13(a) and (b) shows (a sampling of) the directions in $\Omega_{z}$ for monostatic SAR and BISAR with a mobile transmitter, respectively.

Fig. 13(c) shows the a diagram of $\Omega_{z}$ for the case of BISAR with a static transmitter and circular receiver trajectory. To understand this diagram, we note that for a given point $z,\left(\gamma_{R} \widehat{(s)}-\mathbf{z}\right)$ ranges over all directions as the receiver traverses its trajectory. Thus, from (13), $\boldsymbol{\Xi}_{\text {static }}(s, \boldsymbol{z})=\mathrm{J}^{T}\left[\left(\boldsymbol{\gamma}_{T}-\mathbf{z}\right)+\left(\boldsymbol{\gamma}_{R} \widehat{(s)}-\mathbf{z}\right)\right]$ vanishes along certain directions. Thus, $\Omega_{z}$ for BISAR experiment 3 is smaller than that for BISAR experiment 2 , and the reconstructed edges are degraded.

Fig. 11 shows the corresponding plot for BISAR with a static transmitter $\left(\boldsymbol{\gamma}_{T}=(22,0,6.5) \mathrm{km}\right)$ and linear receiver trajectory $\left(\boldsymbol{\gamma}_{R}(s)=\boldsymbol{\gamma}_{l}(s), s \in[0,2 \pi]\right)$. In this case, only a small subset of the possible directions in $\mathbb{R}^{2}$ are contained $\Omega_{\boldsymbol{z}}$, and consequently we reconstruct only a small subset of the edges.

Even more information can be obtained from the set $\Omega_{z}$ as follows. From (13), we see that

$$
\begin{aligned}
0 & \leq\|\boldsymbol{\Xi}(s, z, z)\| \\
& \leq\left[\begin{array}{c}
\left.\| \mathrm{J}\left(z_{1}, z_{2}\right)\right]^{T}\left(\boldsymbol{\gamma}_{T} \widehat{(s)}-\mathbf{z}\right) \| \\
\left.+\| \mathrm{J}\left(z_{1}, z_{2}\right)\right]^{T}\left(\boldsymbol{\gamma}_{R} \widehat{(s)}-\mathbf{z}\right) \|
\end{array}\right] .
\end{aligned}
$$

Thus, for flat topography, $\|\boldsymbol{\Xi}(s, \boldsymbol{z}, \boldsymbol{z})\|$ obtains its maximum when $\left(\boldsymbol{\gamma}_{T} \widehat{(s)}-\mathbf{z}\right)$ and $\left(\boldsymbol{\gamma}_{R} \widehat{(s)}-\mathbf{z}\right)$ are in the same direction, i.e., when we consider a monostatic SAR system. Thus, we will 

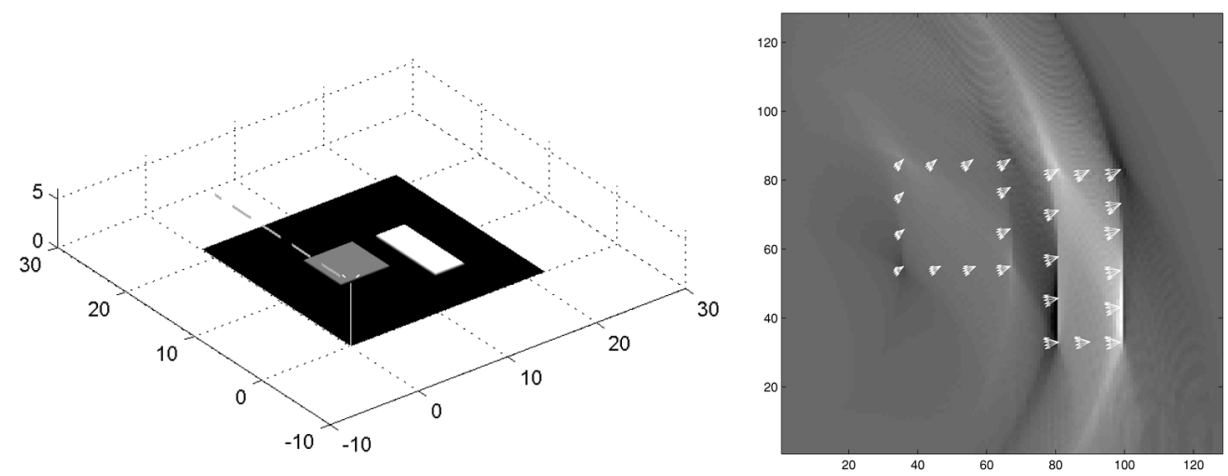

Fig. 11. (Left) 3-D view of (dashed line) linear receiver flight trajectory (axes labels are in $\mathrm{km} \mathrm{s}$ ) and (right) the vectors from $\Omega_{z}$ with fixed frequency $\omega=.1$, for some $z$ on the edges, overlaid on the FBP reconstructed image (axes labels are in pixel number). The data collection manifold includes fewer directions than for the geometry of Fig. 13. Thus, fewer edges are visible, and the frequency content of the reconstructed edges is significantly less than for BISAR with static transmitter and circular receiver flight trajectory.
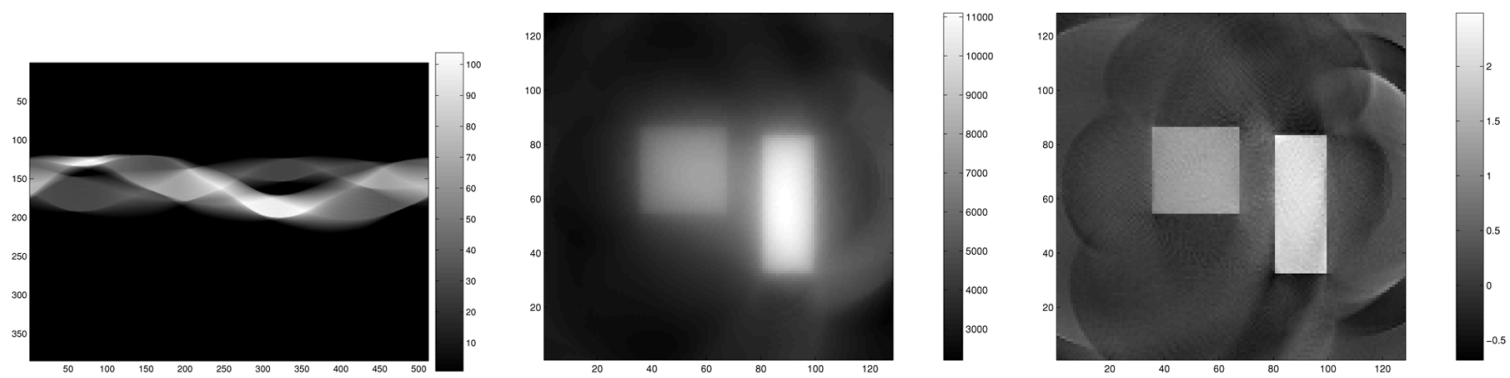

Fig. 12. (Left) Projection data for BISAR for distorted circular transmitter and receiver trajectories, and reconstructed images obtained by (middle) the backprojection and (right) the FBP methods. While the edges are of the right strength and order, as in the circular BISAR case shown in Fig. 9, the distorted circular trajectory leads to more artifacts [which arise from (7) and (8) but are not analyzed in this paper].
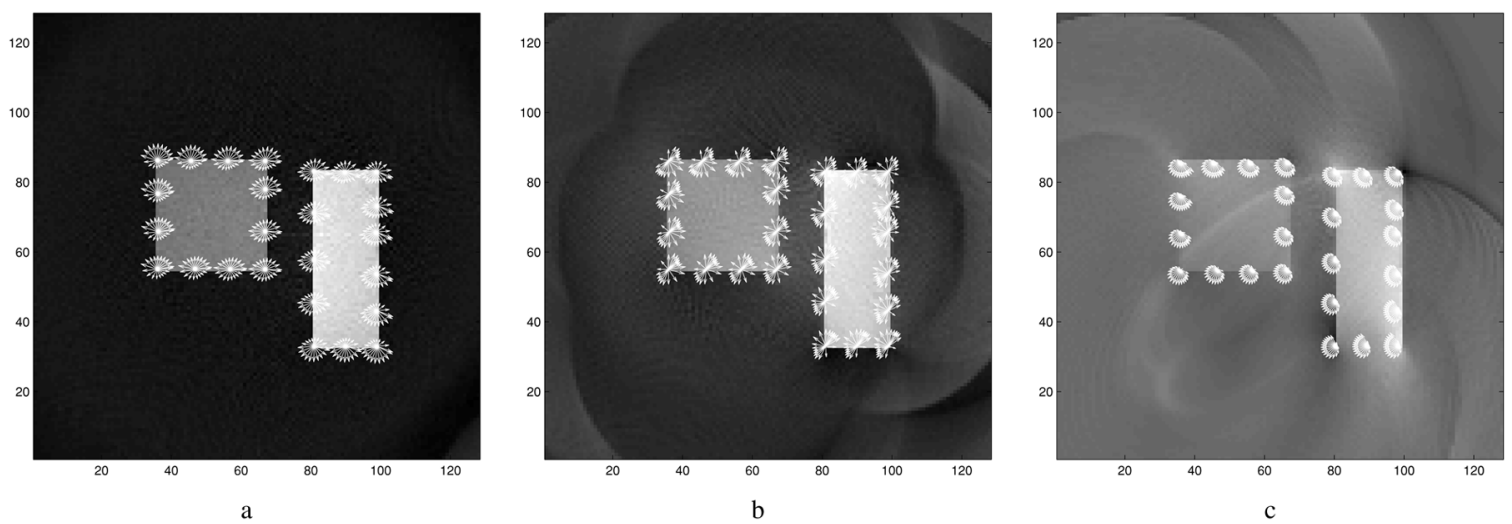

Fig. 13. Vectors in $\Omega_{z}$ at fixed frequency $\omega=.1$, for some $z$ on the edges, overlaid on the reconstructed images for (left) monostatic SAR, (center) BISAR with mobile transmitter, and (right) BISAR with static transmitter. For (left) monostatic SAR and (middle) BISAR with a mobile transmitter, $\Omega_{z}$ includes all directions from $360^{\circ}$; thus, all the edges are visible. For (right) BISAR with static transmitter and circular receiver trajectory, only the directions from a little more than $180^{\circ}$ are included in $\Omega_{z}$. Thus, although all the edges are visible, the frequency content of the reconstructed edges is significantly less than that of the monostatic SAR and BISAR with mobile transmitter.

expect to see sharper edges in the monostatic SAR images than in BISAR images for the same bandwidth. Also, $\Omega_{z}$ for a point near the center of the scene is larger than that for a point near the trajectory, which implies that edges in the center of the scene are sharper than those near the flight path.

\section{CONCLUSION}

In this paper, we developed a new explicit filtered-backprojection-type bistatic synthetic-aperture radar inversion method for arbitrary flight trajectories. The method is based on microlocal analysis and preserves the location, orientation, strength, and order of the visible edges. The method can be extended to multiple bistatic SARs, assuming the receiver can determine which transmitter emitted the waves, in a straightforward manner.

We demonstrated the performance of the inversion method in numerical simulations, which is in correspondence with the theoretical expectations.

We leave for the future the task of applying the techniques of [28] to BISAR. 


\section{APPENDIX \\ STATIONARY PHASE THEOREM}

The stationary phase theorem states [25], [29], [30] that if $a$ is a (possibly complex-valued) smooth function of compact support on $\mathbb{R}^{n}$, and $\phi$ is a real-valued function with only nondegenerate critical points, then as $\omega \rightarrow \infty$

$$
\begin{gathered}
\int \mathrm{e}^{\mathrm{i} 2 \pi \omega \phi(\boldsymbol{x})} a(\boldsymbol{x}) d^{n} \boldsymbol{x} \\
=\sum_{\left\{\boldsymbol{x}^{0}: D \phi\left(\boldsymbol{x}^{0}\right)=0\right\}} \omega^{-n / 2} a\left(\boldsymbol{x}^{0}\right) \frac{\mathrm{e}^{\mathrm{i} 2 \pi \omega \phi\left(\boldsymbol{x}^{0}\right)} e^{i(\pi / 4) \operatorname{sgn} D^{2} \phi\left(\boldsymbol{x}^{0}\right)}}{\sqrt{\left|\operatorname{det} D^{2} \phi\left(\boldsymbol{x}^{0}\right)\right|}} \\
+O\left(\omega^{-n / 2-1}\right) .
\end{gathered}
$$

Here, $D \phi$ denotes the gradient of $\phi, D^{2} \phi$ denotes the Hessian, and sgn denotes the signature of a matrix, i.e., the number of positive eigenvalues minus the number of negative ones.

\section{REFERENCES}

[1] N. J. Willis, Bistatic Radar. Norwood, MA: Artech House, 1991.

[2] W. Goj, Synthetic Aperture Radar and Electronic Warfare. Boston, MA: Artech House, 1993

[3] A. M. Horne and G. Yates, "Bistatic synthetic aperture radar," in Proc. IEEE Radar Conf., Oct. 2002, pp. 6-10.

[4] L. M. H. Ulander and T. Martin, "Bistatic ultrawideband SAR for imaging of ground targets under foliage," in Proc. IEEE Radar Conf., May 2005, pp. 419-423.

[5] D. Munson, J. O'Brien, and W. Jenkins, "A tomographic formulation of spotlight-mode synthetic aperture radar," Proc. IEEE, vol. 71, no. 8, pp. 917-925, Aug. 1983.

[6] O. Arikan and D. C. Munson, Jr., "A tomographic formulation of bistatic synthetic aperture radar," in Proc. ComCon, Oct. 1988, p. 418.

[7] J. C. Curlander and R. N. McDonough, Synthetic Aperture Radar. New York: Wiley, 1991.

[8] G. Franceschetti and R. Lanari, Synthetic Aperture Radar Processing. New York: CRC, 1999.

[9] M. Soumekh, Synthetic Aperture Radar Signal Processing with MATLAB Algorithms. New York: Wiley, 1999.

[10] L. J. Cutrona, "Synthetic aperture radar," in Radar Handbook, M. Skolnik, Ed., 2nd ed. New York: McGraw-Hill, 1990.

[11] C. Elachi, Spaceborne Radar Remote Sensing: Applications and Techniques. Piscataway, NJ: IEEE Press, 1988.

[12] B. Rigling, "Signal processing strategies for bistatic synthetic aperture radar," Ph.D. dissertation, The Ohio State Univ., Columbus, 2003.

[13] S. Nilsson, "Application of fast backprojection techniques for some inverse problems of integral geometry," Ph.D. dissertation, Linköping Studies Sci.Technol., Linköping, Sweden, 1997, Dissertation No. 499.

[14] C. J. Nolan and M. Cheney, "Synthetic aperture inversion," Inv. Probl., vol. 18, pp. 221-236, 2002

[15] C. J. Nolan and M. Cheney, "Synthetic aperture inversion for arbitrary flight paths and non-flat topography," IEEE Trans. Image Process., vol. 12, no. 9, pp. 1035-1043, Sep. 2003.

[16] L. M. H. Ulander, H. Hellsten, and G. Stenström, "Synthetic-aperture radar processing using fast factorized back-projection," IEEE Trans. Aerosp. Electron. Syst., vol. 39, no. 3, pp. 760-776, Jul. 2003.

[17] B. Yazici and M. Cheney, "Synthetic aperture inversion for arbitrary flight paths in the presence of noise and clutter," in Proc. IEEE Int. Radar Conf., May 2005, pp. 806-810.

[18] M. Soumekh, "Wide-bandwidth continuous-wave monostatic/bistatic synthetic aperture radar imaging," in Proc. Int. Conf. Image Processing, Oct. 1998, vol. 3, pp. 361-365.

[19] M. Soumekh, "Bistatic synthetic aperture radar inversion with application in dynamic object imaging," IEEE Trans. Signal Process., vol. 39, no. 9, pp. 2044-2055, Sep. 1991

[20] J. L. Bauck and W. K. Jenkins, "Convolution-backprojection image reconstruction for bistatic synthetic aperture radar," in Proc. IEEE ISCAS, 1989, pp. 1512-1515.
[21] S. Lockwood, A. Brown, and H. Lee, "Backward propagation image reconstruction techniques for bistatic synthetic-aperture radar imaging systems with circular-aperture configurations," in Proc. 35th Asilomar Conf. Signals, Systems, Computers, Nov. 2001, vol. 1, pp. 110-115.

[22] M. Cheney, "Synthetic-aperture assessment of a dispersive surface," Int. J. Imag. Syst. Technol., vol. 14, pp. 28-34, 2004

[23] J. J. Duistermaat, Fourier Integral Operators. Boston, MA: Birkhauser, 1996

[24] F. Treves, Introduction to Pseudodifferential and Fourier Integral Operators, Volumes I and II. New York: Plenum, 1980.

[25] A. Grigis and J. Sjöstrand, Microlocal Analysis for Differential Operators: An Introduction, ser. London Mathematical Society Lecture Note Series. Cambridge, U.K.: Cambridge Univ. Press, 1994, vol. 196.

[26] E. T. Quinto, "Singularities of the X-ray transform and limited data tomography in $r^{2}$ and $r^{3}$," SIAM J. Math. Anal., vol. 24, pp. 1215-1225, 1993.

[27] F. Natterer and F. Wübbeling, Mathematical Methods in Image Reconstruction. Philadelphia, PA: SIAM, 2001

[28] B. Yazıc1, M. Cheney, and C. E. Yarman, "Synthetic aperture inversion in the presence of noise and clutter," Inv. Probl., vol. 22, pp. 1705-1729, 2006.

[29] N. Bleistein and R. A. Handelsman, Asymptotic Expansions of Integrals. New York: Dover, 1986.

[30] V. Guillemin and S. Sternberg, Geometric Asymptotics. Providence, RI: Amer. Math. Soc., 1979.

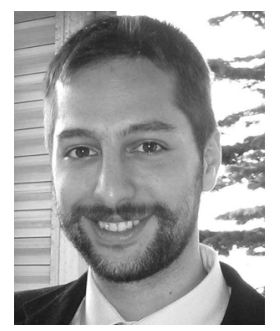

Can Evren Yarman (M'07) received the B.Sc. degree in mathematics from the Middle East Technical University, Ankara, Turkey, the M.Sc. degree in biomedical science from Drexel University, Philadelphia, PA, and the M.Sc. degree in mathematics and the Ph.D. degree in electrical engineering from Rensselaer Polytechnic Institute, Troy, NY, in 2006.

He was a postdoctorate at Rensselaer Polytechnic Insitute from May 2006 to February 2007. Currently, he is a Research Scientist with the Houston Technology Center, WesternGeco-Schlumberger, Houston, TX. His main research interest is inverse problems in imaging.

Birsen Yazıcı (SM'06) received the B.S. degrees in electrical engineering and mathematics from Bogazici University, Istanbul Turkey, in 1988, and the M.S and $\mathrm{Ph} . \mathrm{D}$. degrees from mathematics and electrical engineering from Purdue University, West Lafayette, IN, in 1990 and 1994, respectively.

From September 1994 until 2000, she was a Research Engineer with the General Electric Company Global Research Center, Schenectady, NY. During her tenure in industry, she worked on radar, transportation, industrial, and medical imaging systems. In 2003, she joined Rensselaer Polytechnic Institute, Troy, NY, where she is currently an Assistant Professor in the Department of Electrical, Computer, and Systems Engineering and in the Department of Biomedical Engineering. She holds 11 U.S. patents, and her research interests span the areas of statistical signal processing, inverse problems in imaging, biomedical optics, and radar.

Dr. Yazıcı's work on industrial systems received the second best paper award in 1997 given by the IEEE TRANSACTIONS IN INDUSTRIAL APPLICATIONS. She is also the recipient of the Rensselaer Polytechnic Institute 2007 School of Engineering Research Excellence Award.

Margaret Cheney (M'00) received the B.A. degree in mathematics and physics from Oberlin College, Oberlin, OH, in 1976, and the Ph.D. degree in mathematics from Indiana University, Bloomington, in 1982.

She was a postdoctorate at Stanford University, Stanford, CA, from 1982 to 1984, and an Assistant Professor of mathematics at Duke University, Durham, NC, from 1984 to 1988. Since 1988, she has been at Rensselaer Polytechnic Institute, Troy, NY, where she is a Professor of mathematics. She has held visiting appointments at New York University's Courant Institute (1987-1988), at the Minnesota Institute for Mathematics and Its Applications (1994-1995 and 1997), the Berkeley Mathematical Sciences Research Institute (2001), and the Naval Air Warfare Center Weapons Division (2002). Most of her work has been on the inverse problems that arise in quantum mechanics, acoustics, and electromagnetic theory. 